\title{
Síndrome de vena cava superior en pediatría. Una revisión de la literatura
}

\section{Superior vena cava syndrome in pediatrics. A review of the literature}

\author{
Marco Fidel Sierra-Zúñiga1, Andry Yasmid Mera-Mamián², \\ Eliana Zemanate-Zúñiga ${ }^{3}$
}

\begin{abstract}
Resumen. Las urgencias oncológicas son un reto para el clínico. El síndrome de vena cava superior es una emergencia hemato-oncológica en pediatría, el cual se define como la obstrucción intrínseca o extrínseca de la vena cava superior. La clínica más frecuente en pediatría es la aparición de circulación colateral, edema en esclavina y cianosis facial. El diagnóstico es clínico y debe hacerse lo menos invasivamente posible y de una manera rápida, para iniciar cuanto antes su manejo. Los linfomas, el uso prolongado de catéter venoso central y la cirugía cardiovascular en cardiopatías congénitas, son las principales etiologías. En algunas ocasiones, puede ser necesario un tratamiento empírico para salvar la vida del paciente, mediante el uso de esteroides y radioterapia. El objetivo del presente artículo es revisar y actualizar los aspectos más importantes sobre la etiología, las manifestaciones clínicas, el diagnóstico y el tratamiento del síndrome de vena cava superior en pediatría.
\end{abstract}

Palabras clave: síndrome de la vena cava superior, pediatría, oncología clínica, neoplasia, linfoma, cuidado intensivo.

Abstract. Oncological emergencies are a challenge for the clinician. The superior vena cava syndrome is a hemato-oncological emergency in pediatrics; it is defined as an intrinsic or extrinsic obstruction of the superior vena cava. The most frequent clinical manifestations in children are the appearance of collateral circulation, edema in the body and facial cyanosis. The diagnosis is clinical and should been done as little invasively as possible and without delay to start its management as soon

\footnotetext{
1 Médico, Especialista en Pediatría, Universidad del Cauca. Popayán, Colombia. E-mail: mafisi@unicauca.edu.co.

2 Fisioterapeuta, MSc en Epidemiología en Servicios de Salud. Grupo de Investigación Movimiento y Salud, Facultad de

Fisioterapia, Universidad CES. Medellín, Colombia.

${ }^{3}$ Médica, Especialista en Pediatría, Especialista en Cuidado Intensivo Pediátrico. Profesora Titular, Universidad del Cauca.

Popayán, Colombia.

Conflicto de interés: los autores declaran que no tienen conflicto de interés.

Medicina \& Laboratorio 2021;25:593-603. https://doi.org/10.36384/01232576.451.

Recibido el 1 de abril de 2021; aceptado el 15 de junio de 2021. Editora Médica Colombiana S.A., $2021^{\circ}$.
} 
as possible. Lymphomas, prolonged use of a central venous catheter, and cardiovascular surgery in congenital heart disease are the main etiologies. In some cases, empirical treatment may be necessary to save the patient's life, through the use of steroids and radiotherapy. The aim of this article is to review and update the most important aspects of the etiology, clinical manifestations, diagnosis and treatment of the superior vena cava syndrome in pediatrics.

Keywords: superior vena cava syndrome, pediatrics, clinical oncology, neoplasms, lymphoma, intensive care.

\section{Introducción}

Las urgencias oncológicas representan un reto en la práctica clínica diaria. El síndrome de vena cava superior (SVCS) es una de ellas, debido a la obstrucción del flujo sanguíneo, y a los síntomas respiratorios y neurológicos que se pueden presentar [1-4].

Su historia se remonta al año de 1757, tiempo en el cual William Hunter describió el SVCS en un paciente con un aneurisma sacular de la aorta ascendente, reportado en una autopsia de un paciente [5-8]. Posteriormente, en 1837, Stokes reportó un caso de SVCS secundario a cáncer en pulmón derecho, describiendo una serie de hallazgos clínicos debidos a la compresión de la lesión tumoral sobre la vena cava superior (VCS) [9]. En 1949, Mclntyre y Sykes mencionaron su relación con tumoraciones malignas [7], y en 1954, Schechter encontró que los aneurismas sifilíticos eran una de las principales causas de la época [6]. Más recientemente, el cáncer de pulmón se ha descrito como la causa en aproximadamente el $70 \%$ de los casos presentados en la población general $[6,8]$. En la población pediátrica, los linfomas, el uso prolongado de catéter venoso central y la cirugía cardiovascular en cardiopatías congénitas, son las principales etiologías $[8,10,11]$.
EI SVCS se define como la obstrucción intrínseca o extrínseca de la VCS por diversas causas [1]; generalmente una gran masa en el mediastino anterior que no permite el desarrollo de una circulación colateral efectiva. Cuando se suma la compresión traqueal, se denomina síndrome mediastinal superior [4,12], pero en la población pediátrica, ambos términos suelen ser usados de manera indistinta [12].

El objetivo de este artículo es revisar los aspectos más importantes sobre su etiología, así como la presentación clínica, el diagnóstico y el tratamiento del SVCS en la población pediátrica.

\section{Epidemiología y etiología}

Se estima que aproximadamente 15.000 personas al año en Estados Unidos padecen SVCS [9]. En la población pediátrica, su prevalencia y su incidencia no se conocen con exactitud $[8,13$ 15], debido a la escasa información que se encuentra en la literatura nacional e internacional, la cual se limita en su mayoría a reportes de casos y algunas series de pacientes $[3,4,8,13,16-20]$.

Dentro de las causas más frecuentes en la población adulta está el cáncer broncogénico, responsable de casi el $70 \%$ de los casos [6]. En la población 
pediátrica, los linfomas, el uso prolongado de catéter venoso central y la cirugía cardiovascular en cardiopatías congénitas son las principales etiologías $[3,4,8,10,11,15,21,22]$.

Las causas de SVCS pueden ser intrínsecas o extrínsecas. Las causas intrínsecas (en el interior de la vena), se relacionan con eventos que originen trombosis venosa, como ocurre con los catéteres y los procedimientos cardiovasculares $[4,14,18,23]$, en tanto que las causas extrínsecas (por compresión), se relacionan con tumores mediastinales anteriores como el linfoma, el timoma, el teratoma y el cáncer de tiroides $[12,15,22]$.

En la población pediátrica, se ha descrito que entre el $72 \%$ y el $82 \%$ de las masas mediastinales son malignas, y de estas, entre el $43 \%$ al $47 \%$ son de origen hematológico [11]. Se ha reportado que en los casos de linfoma de Hodgkin, aproximadamente un 30\% presentan compromiso mediastinal, y de estos, un $2 \%$ se manifiestan como un SVCS [11]; por el contrario, en los casos de linfoma no Hodgkin, hasta el $70 \%$ de ellos presentan una masa mediastinal, con el $3 \%$ a $4 \%$ presentando signos y síntomas de un SVCS [11].

Arya y colaboradores en el 2002, Ilevaron a cabo un estudio con 21 pacientes pediátricos (20 de sexo masculino y uno de sexo femenino), encontrando que la leucemia linfoblástica aguda de células $T$ fue la causa más común (57\%) en la etiología del SVCS, seguida del linfoma no Hodgkin (linfoma linfoblástico de células T) [24]; resultados similares han sido reportados en otras series de casos en la literatura mundial $[25,26]$.

En un estudio realizado por Davis y colaboradores, publicado en la Revista
Chilena de Pediatría en 2005, se realizó una revisión retrospectiva de 44 "fichas clínicas" en pacientes menores de 15 años con linfoma. Se encontraron 5 casos asociados a SVCS, 2 con diagnóstico de linfoma de Hodgkin y 3 con linfoma no Hodgkin [13]. Por su parte, Gupta y colaboradores condujeron un estudio entre 2004 y 2006, en el que se incluyeron 18 pacientes pediátricos, encontrando que la causa más frecuente del SVCS fue el linfoma no Hodgkin [21].

El estudio realizado por Kashif y colaboradores en el 2018, incluyó 51 pacientes con sospecha de masa mediastínica maligna. Predominaron los pacientes masculinos (70\%) y el diagnóstico más común fue la leucemia linfoblástica aguda (LLA) de células $T$ (35\%), seguido por el linfoma linfoblástico y el linfoma de Hodgkin, estos dos últimos encontrados en el 15,7\% de los casos [27]. Nossair y colaboradores en el 2018, Ilevaron a cabo una revisión sistemática de la literatura, en la cual se incluyeron 90 reportes de casos y 11 series de casos, para un total de 142 pacientes. Las tres etiologías principales del SVCS fueron causas cardíacas asociadas principalmente con cardiopatía congénita, causas oncológicas asociadas con leucemia, linfoma o tumores sólidos, y causas hematológicas con evidencia de trombo en VCS [20].

Chen y colaboradores en el 2019, publicaron una serie de 40 pacientes menores de 18 años diagnosticados con tumores mediastinales en el Hospital Infantil de la Universidad Médica de China, entre 2001 y 2016. La mayoría de los pacientes eran de sexo masculino (75\%), con una edad media de aparición de 13 años y una tasa de mortalidad del $40 \%$. Los tumores más frecuentes fueron el linfoma (47,5\%), seguido de los tumores de células 
germinales (12,5\%), el neuroblastoma $(12,5 \%)$ y el timoma (7,5\%) [19]. En ese mismo año, Gartrell y colaboradores publicaron un estudio en el cual se realizó una revisión retrospectiva de 330 historias clínicas de pacientes pediátricos diagnosticados con linfomas de Hodgkin $(n=228)$ y linfomas no Hodgkin $(n=102)$ en el St. Jude Children's Research Hospital. Una proporción similar de participantes con linfoma de Hodgkin $(8,7 \%)$ y linfoma no Hodgkin $(7,8 \%)$ desarrollaron tromboembolismo (TE). En el 25,8\% (85/233) de los participantes, hubo compresión de la VCS mayor al $25 \%$, y las probabilidades de desarroIlar un TE cuando la VCS tenía este grado de compresión fue de 2,2 (IC95\% $1,01-4,93 ; p=0,048)$. El estudio concluye que los individuos con una masa mediastínica con un $25 \%$ o más de grado de compresión de la VCS, tenían más probabilidades de desarrollar eventos trombóticos. Este hallazgo destaca un grupo de niños de alto riesgo que pueden beneficiarse de la anticoagulación profiláctica [28].

Finalmente, Ozcan y colaboradores en el 2020, incluyeron en su estudio a 41 pacientes pediátricos. Del total de pacientes con tumores mediastinales, $46 \%$ tenían SVCS (12\% eran de sexo femenino). El diagnóstico incluyó linfoma de Hodgkin en el 37\%, linfoma no Hodgkin en el 32\%, leucemia linfoblástica T aguda en el $21 \%$, y neuroblastoma y sarcoma anaplásico de células redondas en el $2,4 \%$ de los casos [3].

\section{Manifestaciones clínicas}

La fisiopatología del SVCS es explicada por el incremento en la presión de la vena cava superior debido a la obstrucción, lo cual genera disminución del retorno venoso proveniente de la mitad superior del cuerpo hacia la aurícula derecha. La estasis generada por este mecanismo promueve dilatación venosa y extravasación de líquido al tercer espacio, lo que condiciona los signos y síntomas característicos [29].

Dentro de las manifestaciones clínicas más frecuentes se encuentran la aparición de circulación colateral, edema en esclavina y cianosis facial $[3,8,10]$. El incremento de la presión venosa en la región superior del cuerpo, especialmente en cabeza y cuello, causan el edema en esclavina, pudiendo producir a su vez edema cerebral, el cual se puede manifestar como cefalea, confusión y en estados avanzados, con coma $[8,10]$. El edema de cuello y brazos puede producir compromiso funcional de algunas estructuras como la faringe y la laringe, manifestándose clínicamente como tos, ronquera, disnea y disfagia [30,31].

Uno de los signos clásicos es la aparición de distensión de vasos subcutáneos (circulación colateral), como resultado de una disminución en el retorno venoso, llevando a que exista mayor compromiso hemodinámico, evidenciado además por cianosis facial $[8,10,32]$. La obstrucción en la VCS produce un aumento del flujo sanguíneo por medio de colaterales hacia el hemicuerpo inferior [8], generando una sobrecarga de volumen al sistema venoso ácigos y a la vena cava inferior. La presión cervical venosa se encuentra por consiguiente incrementada, y pasa de estar entre $2 \mathrm{mmHg}$ a $8 \mathrm{mmHg}$, que es lo normal, a $20 \mathrm{mmHg}$ a $40 \mathrm{mmHg}$, que es francamente patológica. $\mathrm{El}$ compromiso hemodinámico y la severidad de los síntomas dependen del grado de obstrucción y de la velocidad de instauración del cuadro [10,33,34].

Arya y colaboradores encontraron la fiebre y la tos, seguidas de disnea, 
como los síntomas principales [24], lo cual concuerda con lo mostrado por un estudio llevado a cabo por Ingram y colaboradores, con 24 pacientes pediátricos, en el departamento de hemato-oncología del St. Jude Children's Research Hospital [35]. En el estudio llevado a cabo por Ozcan y colaboradores, el $100 \%$ de los pacientes presentaron edema facial, circulación colateral y tos [3]. Por su parte, Gupta y colaboradores, en el estudio previamente mencionado, describieron el edema en esclavina y la presencia de circulación colateral, como los principales síntomas en esta serie de pacientes [21], en tanto que Kashif y colaboradores, en su serie de pacientes con sospecha de masa mediastínica maligna, observaron fiebre en el $82 \%$ de los casos, y el $58 \%$ cursó con síndrome de dificultad respiratoria; el edema se presentó solo en el $6 \%$ de los casos [27].

Nossair y colaboradores, en su revisión sistemática de la literatura, encontraron que los principales síntomas presentados fueron aquellos relacionados con la obstrucción al flujo sanguíneo (90\%), síntomas respiratorios (70\%) y neurológicos (22\%); el edema y la disnea fueron los más frecuentes. Las principales complicaciones para este estudio fueron el síndrome de efusión pleural y el quilotórax, los cuales ocurrieron en el $55 \%$ de los casos, y se presentó una mortalidad global del 18\%. Las complicaciones se relacionaron con un incremento en la morbimortalidad $(p=0,005)$, en tanto que la presencia de colaterales fue un factor protector $(p=0,007)$, según lo reportado [20].

Por último, Chen y colaboradores encontraron que las presentaciones iniciales de los pacientes evaluados en su estudio, incluyeron dificultad respiratoria $(65 \%)$, tos $(47,5 \%)$ y derrame pleural (54,5\%). El 35\% de los pacientes tuvie- ron como presentación inicial, un SVCS [19].

La tabla 1 muestra el resumen de los principales signos y síntomas encontrados en la literatura.

\section{Diagnóstico}

El diagnóstico debe ser realizado lo más pronto y lo menos invasivamente posible [12]. El enfoque inicial es clínico, teniendo en cuenta los antecedentes del paciente [10]. Dentro de las principales ayudas diagnósticas se encuentran la radiografía de tórax y la tomografía axial computarizada (TAC) de tórax, si son toleradas por el paciente, debido a la posición que se debe adoptar en la toma de estos paraclínicos, que puede aumentar la compresión de estructuras del mediastino e incrementar la dificultad respiratoria y la fatiga $[34,36]$. En los casos en los que no hay evidencia de masa en la radiografía de tórax inicial, pero los hallazgos clínicos sugieran el SVCS, se debe considerar un ecocardiograma [12].

Adicionalmente, se recomienda la realización de pruebas de laboratorio como hemograma, deshidrogenasa láctica, niveles de ácido úrico, electrolitos y evaluación de la función renal, en busca de un síndrome de lisis tumoral que pueda orientar al diagnóstico y tratamiento, además de pruebas especiales como la alfa-fetoproteína y hormona gonadotropina coriónica humana fracción beta ( $\beta$ hCG), en busca de un teratoma y la exclusión de un linfoma [12].

La sedación en el SVCS debe evitarse, pero de ser necesaria, se realizará con extrema precaución con el acompañamiento de personal experto en vía 
Tabla 1. Principales signos y síntomas del síndrome de vena cava superior

\begin{tabular}{|c|c|c|c|}
\hline Autor & Total pacientes & Signos y síntomas & Porcentaje \\
\hline Arya et al [24] & $n=21$ & $\begin{array}{l}\text { Fiebre } \\
\text { Tos } \\
\text { Disnea } \\
\text { Edema en esclavina }\end{array}$ & $\begin{array}{l}90 \% \\
90 \% \\
90 \% \\
62 \%\end{array}$ \\
\hline Ingram et al [35] & $n=24$ & $\begin{array}{l}\text { Tos } \\
\text { Disnea } \\
\text { Disfagia } \\
\text { Ortopnea } \\
\text { Sibilancias }\end{array}$ & $\begin{array}{l}68 \% \\
68 \% \\
63 \% \\
63 \% \\
31 \%\end{array}$ \\
\hline Ozcan et al [3] & $n=41$ & $\begin{array}{l}\text { Edema en esclavina } \\
\text { Circulación colateral } \\
\text { Tos } \\
\text { Disnea }\end{array}$ & $\begin{array}{l}46 \% \\
46 \% \\
46 \% \\
24 \%\end{array}$ \\
\hline Gupta et al [21] & $n=18$ & $\begin{array}{l}\text { Edema en esclavina } \\
\text { Circulación colateral } \\
\text { Tos } \\
\text { Disnea }\end{array}$ & $\begin{array}{c}100 \% \\
100 \% \\
89 \% \\
67 \%\end{array}$ \\
\hline Kashif et al [27] & $\mathrm{n}=51$ & $\begin{array}{l}\text { Fiebre } \\
\text { Disnea } \\
\text { Linfadenopatía } \\
\text { Tos }\end{array}$ & $\begin{array}{l}82 \% \\
58 \% \\
51 \% \\
45 \%\end{array}$ \\
\hline Nossair et al [20] & $n=142$ & $\begin{array}{l}\text { Edema en esclavina } \\
\text { Circulación colateral } \\
\text { Disnea } \\
\text { Tos }\end{array}$ & $\begin{array}{l}82 \% \\
54 \% \\
67 \% \\
15 \%\end{array}$ \\
\hline Chen et al [19] & $\mathrm{n}=40$ & $\begin{array}{l}\text { Disnea } \\
\text { Derrame pleural } \\
\text { Tos }\end{array}$ & $\begin{array}{c}65 \% \\
54,5 \% \\
47,5 \%\end{array}$ \\
\hline
\end{tabular}

aérea, en una unidad de cuidado intensivo pediátrico, teniendo en cuenta 3 aspectos importantes: 1) se puede generar una exacerbación de la obstrucción de la vía aérea preexistente, debido a la relajación de la musculatura lisa a nivel de la tráquea y los bronquios, por el aumento en la compresibilidad secundario al efecto ejercido por la gravedad, a la hora de adoptar el decúbito supino; 2) la disminución en el volumen inspiratorio, debido a la caída en el tono muscular abdominal; $y, 3$ ) el efecto inotrópico negativo y la vasodilatación, que llevan a que el flujo sanguíneo pulmonar disminuya, provocando un descenso en el retorno venoso, empeorando el estado hemodinámico ya deteriorado por la fisiopatología de esta enfermedad [37].

La dificultad en el uso de sedación en esta población suele limitar las ayudas diagnósticas, por lo tanto, siempre se debe determinar el riesgo anestésico de estos pacientes (riesgo de mayor compresión por factor mecánico), aún más si se está pensando en realizar un diagnóstico histológico. Ante un paciente con alto riesgo, se sugiere realizar los procedimientos (biopsia de médula ósea, pleurocentesis, pericar- 
diocentesis, biopsia de ganglio linfático) con anestesia local. Si el riesgo es bajo, la sedación en caso de ser necesaria, podría ser empleada bajo monitorización estricta, como se mencionó previamente, y con la supervisión de un experto en vía aérea [12].

En el estudio de Chen y colaboradores, la obstrucción tumoral de las vías respiratorias, el derrame pleural y el derrame pericárdico fueron las principales causas de inestabilidad cardiopulmonar durante la sedación para procedimientos invasivos [19]. Lo contrario se encontró en la revisión sistemática de la literatura conducida por Ozcan y colaboradores, en donde no hubo asociación entre la anestesia y la morbimortalidad [3].

Establecer el diagnóstico etiológico en algunos pacientes no es tan sencillo. Se debe tener en cuenta que algunas situaciones en particular, pueden empeorar el estado clínico del paciente, llevando a un paro respiratorio; por ejemplo, la posición supina, la sedación y la extubación que pueden convertirse en un reto mayor si son fallidas [12].

\section{Tratamiento}

El tratamiento inicial en el servicio de urgencias es principalmente de apoyo, con elevación de la cabeza, oxígeno, hiperhidratación de ser necesaria, y medidas de soporte [38]. Se debe efectuar el traslado a la unidad de cuidado intensivo pediátrico cuanto antes sea posible por los potenciales riesgos mencionados. En algunas ocasiones, puede ser necesario un tratamiento inicial empírico con el objetivo de salvar la vida del paciente. Al respecto hay dos opciones, a pesar de que ello pueda confundir el diagnóstico: 1) el uso de esteroides con el objetivo de lograr una disminución considerable en la masa tumoral (tumores hematológicos malignos), además de disminuir el edema generado en la vía aérea [21]. Se sugiere prednisolona $60 \mathrm{mg} / \mathrm{m}^{2} /$ día (2 mg/kg/día) o metilprednisolona 48 $\mathrm{mg} / \mathrm{m}^{2} /$ día $(1,6 \mathrm{mg} / \mathrm{kg} /$ día) [12]. En el estudio de Gupta y colaboradores, al igual que en la serie mostrada por Ozcan y colaboradores, se administró dexametasona logrando una reducción en la sintomatología asociada al síndrome $[3,21] ; y, 2)$ la otra opción ante la falla del anterior grupo terapéutico, es la radioterapia $[8,21,39]$. Algunos estudios sugieren combinar radiación y quimioterapia simultáneas para asegurar una regresión rápida de la lesión, sobre todo en los casos en que el SVCS sea causado por tumores altamente sensibles a la quimioterapia como el linfoma y la leucemia, herramientas propias del equipo de hemato-oncología pediátrica [24,39].

En casos de trombosis venosa sintomática sin evidencia de sangrado, se sugiere el inicio de heparina no fraccionada o de bajo peso molecular. Para el uso de heparina no fraccionada, se recomienda iniciar con dosis de $75 \mathrm{U} / \mathrm{kg}$ en bolo, seguidos de una dosis de 28 $\mathrm{U} / \mathrm{kg} / \mathrm{h}$ para niños menores de 1 año, y de $18 \mathrm{U} / \mathrm{kg} / \mathrm{h}$ para niños mayores de 1 año $[12,40]$. Se debe realizar monitoreo con el tiempo de tromboplastina parcial (TTP), el cual debe permanecer entre 60 y 85 segundos, y factor anti$\mathrm{Xa}$, con valores entre 0,3 y $0,7 \mathrm{U} / \mathrm{mL}$. Con heparina de bajo peso molecular, la dosis es de $1 \mathrm{mg} / \mathrm{kg}$ cada 12 horas y monitorizar con niveles de anti- $\mathrm{Xa}$, con valores entre 0,5 y $1 \mathrm{U} / \mathrm{mL}$ [12].

Finalmente, el uso de stent se propone en la literatura como una opción terapéutica en pacientes refractarios al manejo convencional, relacionados con eventos trombóticos y posterior a 
procedimientos cardiovasculares que generen estenosis de la VCS, con resultados prometedores, inclusive superiores a los obtenidos mediante angioplastia con balón [15,22,41].

Se ha tratado de establecer algunos criterios clínicos para determinar el pronóstico y el curso de la enfermedad, dentro de los cuales se mencionan los antecedentes de los pacientes, el tratamiento instaurado y el tipo de masa tumoral maligna que ocasiona el SVCS, sin obtener hasta ahora asociaciones significativas al respecto [42].

En la figura 1 se observa un algoritmo para el abordaje del paciente con SVCS.

\section{Conclusiones}

El SVCS es una emergencia hematooncológica pediátrica. Las principales manifestaciones incluyen la presencia de circulación colateral, aparición de edema en esclavina y cianosis facial, según los datos encontrados en la literatura. El diagnóstico es clínico y debe hacerse de una manera rápida y lo menos invasivamente posible. Algunas ayudas diagnósticas son útiles a la hora de evaluar a nuestros pacientes, como la radiografía de tórax, TAC de tórax y el ecocardiograma. Los estudios histológicos deben realizarse bajo las consideraciones mencionadas cuando la sedación sea requerida.

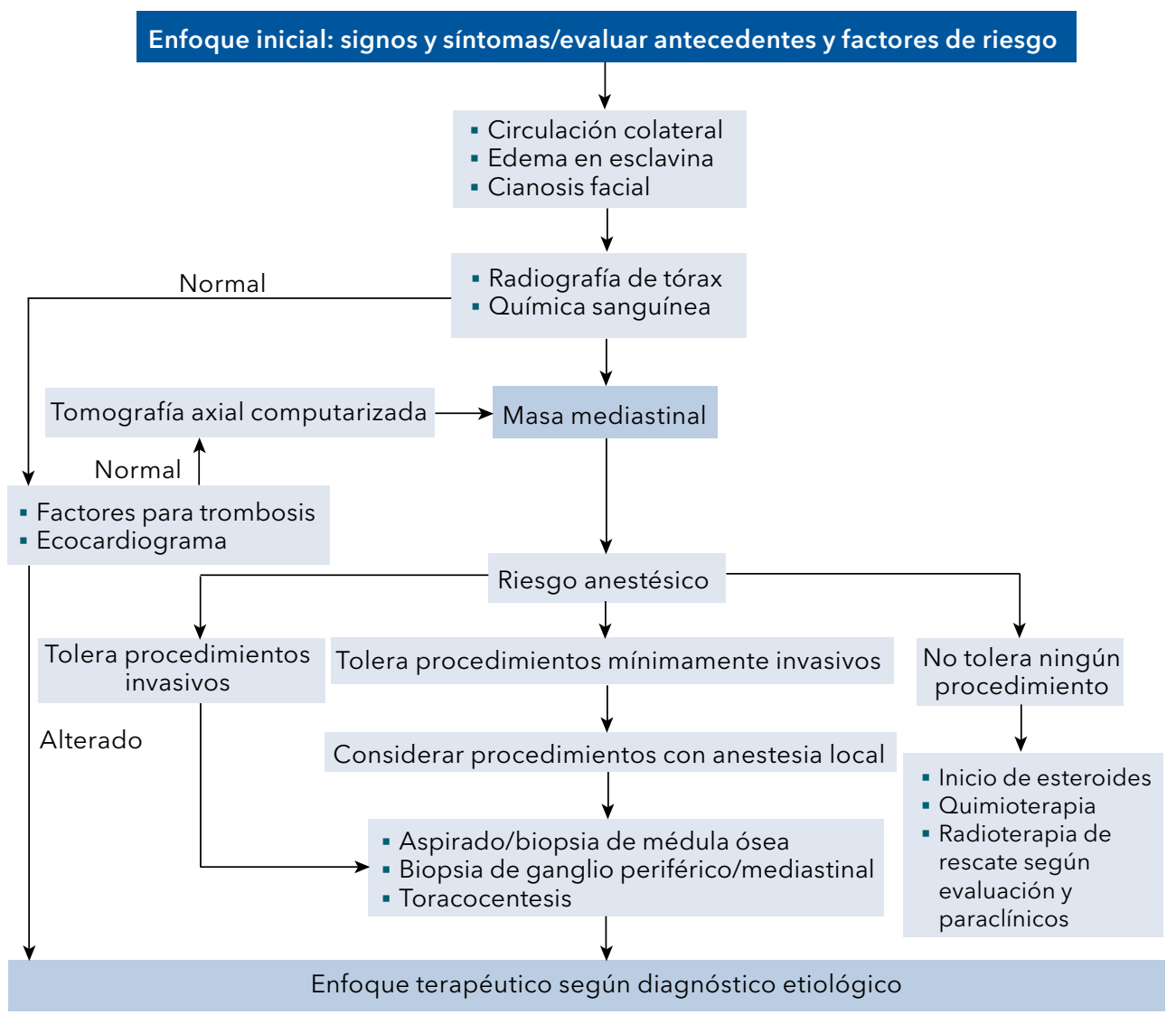

Figura 1. Abordaje de pacientes con síndrome de vena cava superior. 
En el mundo, la incidencia y la prevalencia del SVCS en la población pediátrica son desconocidas. En Colombia, son pocos los estudios publicados al respecto. Esta es una entidad clínica que requiere de una mayor atención en el campo investigativo en el campo investigativo en pediatría, dada su alta complejidad. Por tal razón es necesario incrementar el reporte de los casos identificados y tratados, de tal modo que se pueda sumar evidencia que permita direccionar las mejores estrategias de diagnóstico y tratamiento oportuno, para evitar así complicaciones e incidir en su morbimortalidad.

\section{Agradecimientos}

A la Universidad del Cauca y a su Departamento de Pediatría.

\section{Referencias}

1. Alvarado-Arce E, Odio-Cortés C. Síndrome de vena cava superior: una emergencia médico quirúrgica. Rev Clin Esc Med 2015;5:11-21.

2. Talapatra K, Panda S, Goyle S, Bhadra K, Mistry R. Superior vena cava syndrome: A radiation oncologist's perspective. J Cancer Res Ther 2016;12:515-519. https://doi. org/10.4103/0973-1482.177503.

3. Ozcan A, Unal E, Karakukcu M, Coskun A, Ozdemir MA, Patiroglu T. Vena cava superior syndrome in the children with mediastinal tumors: Single-center experience. North Clin Istanb 2020;7:255-259. https://doi. org/10.14744/nci.2019.46354.

4. Handa A, Nozaki T, Makidono A, Okabe T, Morita Y, Fujita K, et al. Pediatric oncologic emergencies: Clinical and imaging review for pediatricians. Pediatr Int 2019;61:122-139. https://doi.org/10.1111/ped.13755.

5. Hunter $\mathbf{W}$, Johnston $\mathbf{W}$. The history of an aneurysm of the aorta, with some remarks on aneurysms in general. Volumen 1, Medical ob- servations and inquiries. London, UK: William Johnston; 1757. p. 357.

6. Lanuti M, De Delva PE, Gaissert HA, Wright CD, Wain JC, Allan JS, et al. Review of superior vena cava resection in the management of benign disease and pulmonary or mediastinal malignancies. Ann Thorac Surg 2009;88:392-397. https://doi.org/10.1016/j. athoracsur.2009.04.068.

7. Leo F, Bellini R, Conti B, Delledonne V, Tavecchio L, Pastorino U. Superior vena cava resection in thoracic malignancies: does prosthetic replacement pose a higher risk? Eur J Cardiothorac Surg 2010;37:764-769. https://doi. org/10.1016/j.ejcts.2009.10.024.

8. O'Brien RT, Matlak ME, Condon VR, Johnson DG. Superior vena cava syndrome in children. West J Med 1981;135:143-147.

9. Wudel LJ, Jr., Nesbitt JC. Superior vena cava syndrome. Curr Treat Options Oncol 2001;2:7791. https://doi.org/10.1007/s11864-001-0019-3.

10. Wilson LD, Detterbeck FC, Yahalom J. Superior vena cava syndrome with malignant causes. N Engl J Med 2007;356:1862-1869. https://doi. org/10.1056/NEJMcp067190.

11. Freud E, Ben-Ari J, Schonfeld T, Blumenfeld A, Steinberg R, Dlugy E, et al. Mediastinal tumors in children: a single institution experience. Clin Pediatr (Phila) 2002;41:219-223. https:// doi.org/10.1177/000992280204100404.

12. Freedman JL, Rheingold SR. Chapter 32 - Management of Oncologic Emergencies. In: Lanzkowsky P, Lipton JM, Fish JD, eds. Lanzkowsky's Manual of Pediatric Hematology and Oncology (Sixth Edition). San Diego: Academic Press; 2016. p. 605-619. https://doi.org/10.1016/ B978-0-12-801368-7.00032-6.

13. Davis GM, Zolezzi RP, Zumelzu DN. Síndrome de vena cava superior: Una emergencia oncológica en niños con linfoma. Revisión de 5 casos. Rev Chil Pediatr 2005;76:507-512.

14. Ji D, Gill AE, Ermentrout RM, Hawkins CM. Thrombogenic superior vena cava syndrome from long-standing central venous access in a 5-year-old patient treated with balloon-expandable stents. J Radiol Case Rep 2018;12:15-22. https://doi.org/10.3941/jrcr.v12i4.3339.

15. Tzifa A, Marshall AC, McElhinney DB, Lock JE, Geggel RL. Endovascular treatment for 
superior vena cava occlusion or obstruction in a pediatric and young adult population: a 22-year experience. J Am Coll Cardiol 2007;49:1003-1009. https://doi.org/10.1016/j. jacc.2006.10.060.

16. Yilmaz O, Karabag K, Keskin Yildirim Z, Calik $\mathbf{M}$, Kilic O. Advanced stage T-cell non-hodgkin lymphoma in an 11-month-old infant and related superior vena cava syndrome: Importance of transthoracic echocardiography. Malays J Med Sci 2014;21:62-65.

17. Rizvi I, Zaman S, Zaidi N, Ashraf SM, Kumar A, Gupta A, et al. Superior vena cava syndrome caused by Hodgkin's lymphoma in an adolescent girl. BMJ Case Rep 2012;2012:bcr0120125487. https://doi. org/10.1136/bcr.01.2012.5487.

18. Thapa S, Terry PB, Kamdar BB. Hemodialysis catheter-associated superior vena cava syndrome and pulmonary embolism: a case report and review of the literature. BMC Res Notes 2016;9:233-233. https://doi.org/10.1186/ s13104-016-2043-1.

19. Chen $\mathrm{CH}$, Wu KH, Chao YH, Weng DF, Chang JS, Lin CH. Clinical manifestation of pediatric mediastinal tumors, a single center experience. Medicine (Baltimore) 2019;98:e16732. https:// doi.org/10.1097/md.0000000000016732.

20. Nossair F, Schoettler P, Starr J, Chan AKC, Kirov I, Paes B, et al. Pediatric superior vena cava syndrome: An evidence-based systematic review of the literature. Pediatr Blood Cancer 2018;65:e27225. https://doi.org/10.1002/ pbc. 27225.

21. Gupta V, Ambati SR, Pant $P$, Bhatia B. Superior vena cava syndrome in children. Indian J Hematol Blood Transfus 2008;24:28-30. https://doi. org/10.1007/s12288-008-0020-0.

22. Aldoss O, Arain N, Menk J, Kochilas L, Gruenstein D. Endovascular stent provides more effective early relief of SVC obstruction compared to balloon angioplasty. Catheter Cardiovasc Interv 2014;83:e272-276. https:// doi.org/10.1002/ccd.24413.

23. Zouari M, Ben Dhaou M. Superior vena cava syndrome secondary to the placement of a central venous catheter in a neonate. Pan Afr Med J 2015;20:305-305. https://doi.org/10.11604/ pamj.2015.20.305.6118.
24. Arya LS, Narain S, Tomar S, Thavaraj V, Dawar R, Bhargawa M. Superior vena cava syndrome. Indian J Pediatr 2002;69:293-297. https://doi. org/10.1007/bf02723212.

25. Yellin A, Mandel M, Rechavi G, Neuman $Y$, Ramot B, Lieberman Y. Superior vena cava syndrome associated with lymphoma. Am J Dis Child 1992;146:1060. https://doi.org/10.1001/ archpedi.1992.02160210062022.

26. Issa PY, Brihi ER, Janin Y, Slim MS. Superior vena cava syndrome in childhood: report of ten cases and review of the literature. Pediatrics 1983;71:337-341.

27. Kashif RU, Faizan M, Anwar S. Pediatric malignant mediastinal masses. J Coll Physicians Surg Pak 2019;29:258-262. https://doi. org/10.29271/jcpsp.2019.03.258.

28. Gartrell J, Kaste SC, Sandlund JT, Flerlage J, Zhou Y, Cheng $\mathbf{C}$, et al. The association of mediastinal mass in the formation of thrombi in pediatric patients with non-lymphoblastic lymphomas. Pediatr Blood Cancer 2020;67:e28057. https://doi.org/10.1002/pbc.28057.

29. Pech-Alonso B, Arredondo-Ruiz P, GonzálezGalván LM, Fermín-Hernández C. Síndrome de la vena cava superior: diagnóstico y tratamiento. Med Interna Mex 2018;34:403-411.

30. McGarry RC. Superior vena cava obstruction due to prostate carcinoma. Urology 2000;55:436. https://doi.org/10.1016/s0090-4295(99)00543-9.

31. Montalbán C, Moreno MA, Molina JP, Hernanz I, Bellas C. Metastatic carcinoma of the prostate presenting as a superior vena cava syndrome. Chest 1993;104:1278-1280. https:// doi.org/10.1378/chest.104.4.1278.

32. Pinto-Marín A, González-Barón M. [Superior vena cava syndrome]. Med Clin (Barc) 2009;132:195-199. https://doi.org/10.1016/j. medcli.2008.06.012.

33. Yu JB, Wilson LD, Detterbeck FC. Superior vena cava syndrome--A proposed classification system and algorithm for management. J Thorac Oncol 2008;3:811-814. https://doi. org/10.1097/JTO.0b013e3181804791.

34. Wan JF, BezjakA. Superior vena cava syndrome. Emerg Med Clin North Am 2009;27:243-255. https://doi.org/10.1016/j.emc.2009.01.003.

35. Ingram L, Rivera GK, Shapiro DN. Superior vena cava syndrome associated with child- 
hood malignancy: analysis of 24 cases. Med Pediatr Oncol 1990;18:476-481. https://doi. org/10.1002/mpo.2950180608.

36. Rice TW, Rodriguez RM, Barnette R, Light RW. Prevalence and characteristics of pleural effusions in superior vena cava syndrome. Respirology 2006;11:299-305. https://doi. org/10.1111/j.1440-1843.2006.00841.x.

37. Brenan R. Oncologic emergencies. Rotation in pediatric Oncology. Memphis, United States: St. Jude Childrens Research Hospital; 2011.

38. Zimmerman S, Davis M. Rapid fire: Superior vena cava syndrome. Emerg Med Clin North Am 2018;36:577-584. https://doi.org/10.1016/j. emc.2018.04.011.

39. Davenport D, Ferree C, Blake D, Raben M. Response of superior vena cava syndrome to radiation therapy. Cancer 1976;38:1577-1580. https://doi. org/10.1002/1097-0142(197610)38:4<1577::AIDCNCR2820380422>3.0.CO;2-K

40. Dabbous MK, Sakr FR, Malaeb DN. Anticoagulant therapy in pediatrics. J Basic Clin Pharm 2014;5:27-33. https://doi.org/10.4103/09760105.134947.

41. Gómez JJ, Vallejo E, Palma MA, Rojas JP. Manejo exitoso con stent en un prematuro con síndrome de vena cava superior: Reporte de caso. Rev Colomb Cardiol 2012;19:208-211.

42. Chan RC, Chan YC, Cheng SW. Mid- and longterm follow-up experience in patients with malignant superior vena cava obstruction. Interact Cardiovasc Thorac Surg 2013;16:455-458. https://doi.org/10.1093/icvts/ivs562. 